\author{
SERIES “THEMATIC REVIEW SERIES ON PULMONARY \\ REHABILITATION" \\ Edited by M.A. Spruit and E.M. Clini \\ Number 1 in this Series
}

\title{
Practical recommendations for exercise training in patients with COPD
}

\author{
Rainer Gloeckl*, Blagoi Marinov ${ }^{\#}$ and Fabio Pitta
}

\begin{abstract}
The aim of this article was to provide practical recommendations to healthcare professionals interested in offering a pulmonary rehabilitation programme for patients with chronic obstructive pulmonary disease (COPD). The latest research findings were brought together and translated into clinical practice. These recommendations focus on the description of useful assessment tests and of the most common exercise modalities for patients with COPD. We provide specific details on the rationale of why and especially how to implement exercise training in patients with COPD, including the prescription of training mode, intensity and duration, as well as suggestions of guidelines for training progression.
\end{abstract}

KEYWORDS: Chronic obstructive pulmonary disease, exercise training, guidelines, pulmonary rehabilitation, recommendations

$\mathbf{T}$ he recent statement on pulmonary rehabilitation of the American Thoracic Society/ European Respiratory Society (ATS/ERS) describes pulmonary rehabilitation as a "comprehensive intervention based on a thorough patient assessment followed by patient-tailored therapies which include, but are not limited to, exercise training, education and behaviour change, designed to improve the physical and emotional condition of people with chronic respiratory disease and to promote the long-term adherence to health-enhancing behaviour" (personal communication; M. Spruit, CIRO+, Horn, the Netherlands).

Pulmonary rehabilitation has been demonstrated to improve exercise tolerance, reduce symptoms of dyspnoea and increase health-related quality of life. Therefore, pulmonary rehabilitation is regarded as one of the most effective nonpharmacological treatments in patients with chronic obstructive pulmonary disease (COPD) $[1,2]$. This article guides the reader through some of the key points on how to set up a pulmonary rehabilitation programme for patients with COPD. The common process of pulmonary rehabilitation, consisting of assessment, intervention and outcome, will be revealed and discussed. Since exercise training is regarded as one of the cornerstones of pulmonary rehabilitation, based on the highest scientific evidence levels (table 1), this article provides an overview on different methods for assessing patients' exercise capacity, as well as introducing the most common and some new exercise modalities applied to patients with COPD. Special emphasis is placed on practical recommendations that can be directly applied in clinical practice.

\section{PATIENTS' ASSESSMENT}

Patients with COPD may respond to exercise training in different ways compared to healthy subjects since the determinants of exercise limitation appear to be widely multi-factorial. Such factors may include gas exchange abnormalities, dynamic lung hyperinflation, insufficient energy supply to the peripheral and respiratory muscles, morphological alterations in leg and diaphragm muscle fibres and reduced functional metabolic capacities [4,5]. Exercise performance will be limited by the weakest component(s) of this physiological chain.

A good way to start the implementation of a pulmonary rehabilitation programme is to make sure that patients undergo an adequate assessment of their physical capacity. When conducting assessment tests it is important to find out the main causes of exercise limitation. The information
AFFILIATIONS

*Dept of Respiratory Medicine and Exercise Therapy, Schoen Klinik Berchtesgadener Land, Schoenau am Koenigssee, Germany,

"Pathophysiology Dept, Medical University of Plovdiv, Pulmonary Function Laboratories, Plovdiv, Bulgaria, and

"Dept of Physiotherapy, Laboratory of

Research in Respiratory

Physiotherapy, State University of

Londrina, Londrina, Brazil.

CORRESPONDENCE

R. Gloeckl

Dept of Respiratory Medicine and Exercise Therapy

Schoen Klinik Berchtesgadener Land Malterhoeh 1

Schoenau am Koenigssee 83471

Germany

E-mail: rainer.gloeckl@gmx.de

Received:

Feb 152013

Accepted after revision:

March 222013

PROVENANCE

Submitted article, peer reviewed.

European Respiratory Review

Print ISSN 0905-9180

Online ISSN 1600-0617 


\begin{tabular}{|c|c|c|}
\hline TABLE 1 & \multicolumn{2}{|c|}{$\begin{array}{l}\text { Benefits and evidence levels of pulmonary } \\
\text { rehabilitation outcomes in chronic obstructive } \\
\text { pulmonary disease (COPD) }\end{array}$} \\
\hline Benefits & & Evidence \\
\hline \multicolumn{2}{|c|}{ Improves exercise capacity } & A \\
\hline \multicolumn{2}{|c|}{ Reduces the perceived intensity of breathlessness } & A \\
\hline \multicolumn{2}{|c|}{ Improves health-related quality of life } & A \\
\hline \multicolumn{2}{|c|}{$\begin{array}{l}\text { Reduces the number of hospitalisations and } \\
\text { hospital days }\end{array}$} & A \\
\hline \multicolumn{2}{|c|}{$\begin{array}{l}\text { Reduces anxiety and depression associated } \\
\text { with COPD }\end{array}$} & A \\
\hline \multicolumn{2}{|c|}{$\begin{array}{l}\text { Strength and endurance training of the upper } \\
\text { limbs improves arm function }\end{array}$} & B \\
\hline \multicolumn{2}{|c|}{$\begin{array}{l}\text { Benefits extend well beyond the immediate } \\
\text { period of training }\end{array}$} & B \\
\hline \multicolumn{2}{|c|}{ Improves survival } & B \\
\hline \multicolumn{2}{|c|}{$\begin{array}{l}\text { Respiratory muscle training can be beneficial, } \\
\text { especially when combined with general exercise training }\end{array}$} & C \\
\hline \multicolumn{3}{|c|}{$\begin{array}{l}\text { Category A: randomised controlled trials, rich body of data; Category B: } \\
\text { randomised controlled trials, limited body of data; Category C: nonrandomised } \\
\text { trials or observational studies. Reproduced from [3] with permission from the } \\
\text { publisher. }\end{array}$} \\
\hline
\end{tabular}

provided by these tests is helpful in designing an individually tailored exercise programme. Continuous and interval endurance training, as well as strength training, may be regarded as the major exercise components. The application of additional exercise methods, for example breathing exercises, inspiratory muscle training, neuromuscular electrical stimulation or whole body vibration training, may also be useful techniques which will be discussed later.

\section{Assessment tests}

Exercise tolerance can be assessed by a cardiopulmonary exercise test using either cycle ergometry or a treadmill, measuring a number of physiological variables, including peak oxygen uptake $\left(V^{\prime} \mathrm{O}_{2}\right)$, peak heart rate and peak work performance. A less complex approach is to use a self-paced, timed walking test (e.g. 6-min walk test (6MWT)). This test requires at least one practice test before data can be interpreted. Shuttle walking tests are also a useful option. They provide more in-depth comprehensive information than an entirely self-paced test, but are easier to perform than a cardiopulmonary exercise test [6]. Additional assessment of muscle strength of the lower and upper extremities also adds important information and provides a comprehensive insight into patients' limitations derived from extrapulmonary manifestations of COPD.

\section{Cardiopulmonary exercise testing}

Incremental cycle ergometry

This test is widely used in clinical practice. A progressively increasing work rate protocol enables rapid acquisition of diagnostic data. Because the response of some of the major interesting variables, such as minute ventilation, $V^{\prime} \mathrm{O}_{2}$ and carbon dioxide uptake, lags behind changes in work rate, it is important to employ a protocol in which work rate increases at a constant rate. For the same reason it is useful to start the testing phase from a baseline of unloaded pedalling at $0 \mathrm{~W}$. The ATS/ American College of Chest Physicians (ACCP) statement on exercise testing recommends starting with a resting phase of $3 \mathrm{~min}$ followed by $3 \mathrm{~min}$ of unloaded pedalling before the incremental phase [7]. The intensity should then be increased every minute by $5-25 \mathrm{~W}$ until the patient reaches volitional exhaustion. Alternatively, a ramp protocol could be used, usually increasing intensity every few seconds. However, the total increment per minute in the ramp protocol should be similar to that of the previous protocol showing a similar metabolic response [8]. In general, exercise tests in which the incremental phase is completed between 8-12 min are efficient and provide useful diagnostic information. Outcome parameters from the test such as peak work rate, peak heart rate or peak $V^{\prime} \mathrm{O}_{2}$ can be used to derive exercise intensities for an endurance training protocol on a cycle ergometer.

\section{Constant work rate test}

This type of test protocol is gaining popularity due to its clinical relevance and its more sensitive response to therapeutic interventions in comparison with an incremental test protocol [9]. Before conducting a constant work rate test it is necessary to perform a maximal cardiopulmonary (incremental) exercise test. For the constant work rate test, the patient cycles at $\sim 70 \%$ of their peak work rate until exhaustion. The time the patient is able to sustain cycling is regarded as the outcome parameter.

\section{6-min walk test}

The $6 \mathrm{MWT}$ is probably the most popular field walking test used for patients with respiratory disorders. It evaluates the global and integrated responses of all systems involved during exercise, including the pulmonary and cardiovascular system, neuromuscular units and muscle metabolism. It is generally believed that the self-paced 6MWT assesses the sub-maximal level of functional capacity, although reaching high levels of cardiopulmonary stress. The 6-min walking distance (6MWD) seems to better reflect the function exercise level for daily physical activities than maximal incremental tests [10]. Furthermore, oxygen desaturation during the 6MWT may also reflect oxygen desaturation during the patients' activities of daily living [11].

The 6MWT requires a 30-m hallway but no exercise equipment. The test measures the distance that a patient is able to walk quickly on a flat, hard surface in a period of 6 min back and forth around cones. A rigorous standardisation of the test procedure [12], especially concerning the verbal communication before and during the test, is very important to minimise a potential bias by the tester. Some of the basic instructions that should be mentioned before the test include the patient being encouraged to "...walk as far as possible for $6 \mathrm{~min}^{\prime \prime}$ and that they "...probably will get out of breath or become exhausted". Therefore, the patient is "permitted to slow down, to stop and to rest if necessary". During the test the tester should only use standard phrases of encouragement every minute with an even tone of voice; for example, "You are doing well. You have 3 minutes to go". At the beginning and the end of the test the patient's oxygen saturation, heart rate, perceived dyspnoea 
and leg fatigue on a Borg scale are generally documented, as well as the total distance walked (in metres) during the test.

\section{Incremental shuttle walking tests}

The incremental shuttle walking test (ISWT) is also a field walking test; however, it differs from the 6MWT as it uses an audio signal from a CD player to determine the walking pace of the patient back and forth on a 10-m course [6]. The walking speed increases every minute, and the test ends when the patient is not able to reach the turnaround point within the required time. The distance walked is noted as a primary outcome parameter. The power output is similar to a symptom-limited, maximal, incremental treadmill test. An advantage of the ISWT is that it shows a better correlation with peak $V^{\prime} \mathrm{O}_{2}$ than the $6 \mathrm{MWD}$ as this test determines the maximum exercise capacity. Disadvantages include less widespread use and more potential for cardiovascular risks, since it evokes maximal exertion from the patients.

A related variation of the ISWT is the endurance shuttle walking test (ESWT). Patients are asked to walk at a speed equivalent to $85 \%$ of the peak speed achieved during the ISWT until exhaustion [13]. Walking time is taken as outcome. The ESWT shows major improvements following pulmonary rehabilitation and is more sensitive to changes than the field tests of maximal capacity [14].

\section{Sit-to-stand tests}

Another simple test procedure to determine functional exercise capacity is a sit-to-stand test. The test involves either the number of sit-to-stand repetitions from a standard chair within $30 \mathrm{~s}$, respectively $60 \mathrm{~s}$, or quantifies the time that a patient needs to perform, for example, five repetitions in a row. These tests may also determine functional status as easily as the $6 \mathrm{MWT}$ in regard to neurophysiologic effectiveness [15, 16]. Moreover a recent study has even shown a strong correlation between the result from a sit-to-stand test and mortality [17].

\section{Peripheral muscle strength testing}

As COPD is a disease with extrapulmonary, systemic manifestations such as muscle dysfunction [18], it is also important to assess peripheral muscle function. Muscle strength is usually expressed as the maximal voluntary isometric force of a muscle. As a reflection of lower limb strength, the quadriceps femoris muscle is, mostly, tested. Important requirements for a valid measurement are a proper fixation of the patient so that they cannot make any evasion movement; compensatory movements, and strong encouragement to ensure the highest possible muscle contraction. Devices such as an isokinetic dynamometer, a special chair using a strain gauge fixed at the ankle, or hand-held dynamometers can be used to determine muscle strength. Handgrip force, measured by a handgrip dynamometer, can be considered as an indicator for upper extremity strength. All strength measurements can be expressed in relation to normal values $[19,20]$.

\section{IMPLEMENTING AN EXERCISE TRAINING PROGRAMME IN PULMONARY REHABILITATION}

The "conventional" modalities used to exercise patients with COPD participating in pulmonary rehabilitation programmes mainly include endurance (continuous or interval) training and strength training.

\section{Endurance training}

Endurance training is probably the most common exercise modality in patients with COPD. The main objective of endurance training is to improve aerobic exercise capacity as aerobic activities are part of many everyday tasks in these patients. It has been shown that endurance training also improves peripheral muscle function in patients with COPD [21]. In addition, there is some evidence that high-intensity endurance training induces greater physiological benefits than lower-intensity exercise [22]. However, most patients with severe COPD are not able to sustain high-intensity exercise due to serious symptoms, such as dyspnoea and fatigue [23]. Therefore, alternative exercise protocols, such as interval training, have gained increasing interest especially in patients with advanced COPD.

\section{Continuous versus interval training}

Historically, the rationale for interval training included the ability to impose high-power bursts of exercise on peripheral muscles without overloading the cardio-respiratory system. As outlined previously, people with COPD respond to training in a different way to healthy subjects as they are restricted by the complex interaction of different determinants of exercise limitation.

A recent systematic review included eight randomised controlled trials with a total of 388 COPD patients and compared the effects of continuous and interval training in a meta-analysis (mean forced expiratory volume in $1 \mathrm{~s} 33-55 \%$ predicted) [24]. The authors summarised that both exercise modalities led to comparable improvements in exercise capacity and health-related quality of life. Continuous and interval endurance training also significantly improved the capillary-to-fibre ratio as well as the fibre-type distribution within the vastus lateralis muscle in similar amounts. Accordingly, there is a significant reduction in the proportion of anaerobic fast-twitch (type $\mathrm{IIb}$ ) muscle fibres following both training regimes yielding a higher percentage of aerobic slowtwitch (type I) muscle fibres [21]. The benefits in terms of improving exercise tolerance, quality of life and muscle fibre morphology and typology were comparable across patients with COPD in Global Initiative of Chronic Obstructive Lung Disease (GOLD) stages II, III and IV [25].

Nevertheless, in patients with very severe COPD there is evidence that interval training is associated with fewer symptoms of dyspnoea during exercise and fewer unintended breaks $[26,27]$. Therefore patients with severe COPD may markedly increase the total exercise duration with significantly lower metabolic and ventilatory stress, as well as lower rates of dynamic hyperinflation when performing interval training compared to continuous training [28].

Although interval training consists of a sequence of on-and-off high-intensity muscular loads, its tolerability in the context of perceived respiratory and peripheral muscle discomfort has been shown to be better than that of constant load exercise [29].

This may indicate a better feasibility of interval training protocols, especially in patients with severe airflow obstruction. In general, many patients are frustrated by the burden of physical limitation in daily life. To avoid frustration during exercise training it may be important to offer exercise protocols that are feasible to each specific patient. It is speculated that 
TABLE 2 Practical recommendations for the implementation of continuous and interval endurance training programmes

\begin{tabular}{|c|c|c|}
\hline Frequency & 3-4 days $\cdot$ week $^{-1}$ & 3-4 days $\cdot$ week $^{-1}$ \\
\hline \multirow[t]{3}{*}{ Mode } & Continuous & Interval modes: \\
\hline & & $30 \mathrm{~s}$ of exercise, $30 \mathrm{~s}$ of rest or \\
\hline & & $20 \mathrm{~s}$ of exercise, $40 \mathrm{~s}$ of rest \\
\hline \multirow[t]{3}{*}{ Intensity } & Initially $60-70 \%$ of PWR & Initially $80-100 \%$ of PWR for the first three to four sessions \\
\hline & Increase work load by $5-10 \%$ as tolerated & Increase work load by $5-10 \%$ as tolerated \\
\hline & Progressively try to reach $\sim 80-90 \%$ of baseline PWR & Progressively try to reach $\sim 150 \%$ of baseline PWR \\
\hline \multirow[t]{2}{*}{ Duration } & Initially $10-15$ min for the first three to four sessions & Initially $15-20 \mathrm{~min}$ for the first three to four sessions \\
\hline & Progressively increase exercise duration to $30-40 \mathrm{~min}$ & $\begin{array}{l}\text { Progressively increase exercise duration to } 45-60 \mathrm{~min} \\
\text { (including resting time) }\end{array}$ \\
\hline Perceived exertion & $\begin{array}{l}\text { Try to aim for a perceived exertion on the 10-point Borg scale } \\
\text { of } 4 \text { to } 6\end{array}$ & $\begin{array}{l}\text { Try to aim for a perceived exertion on the 10-point Borg scale } \\
\text { of } 4 \text { to } 6\end{array}$ \\
\hline Breathing technique & $\begin{array}{l}\text { Suggest pursed-lip breathing or the use of PEP devices to prevent } \\
\text { dynamic hyperinflation and to reduce breathing frequency }\end{array}$ & $\begin{array}{l}\text { Suggest pursed-lip breathing or the use of PEP devices to prevent } \\
\text { dynamic hyperinflation and to reduce breathing frequency }\end{array}$ \\
\hline
\end{tabular}

PWR: peak work rate; PEP: positive expiratory pressure. Adapted from [30]

this could reveal a psychological advantage to improve the patients' motivation and maybe also increase long-term adherence to exercise training programmes. Nevertheless, especially older, patients with COPD initially have to familiarise themselves with this exercise mode and resting intervals in order to follow the right sequence of work and rest intervals for the required period.

An easy approach to target training intensity for continuous and interval endurance training on a bicycle would be to derive exercise intensity from a certain percentage of the peak work load (e.g. 70\%). To further adjust cycling load to an effective, as well as feasible, intensity the patients' perceived exertion on the modified Borg scale (0-10) should be aimed at 4 to 6 . Table 2 shows some practical recommendations for the implementation of continuous and interval endurance training programmes.

So how to find the right endurance training protocol for your patient? Table 3 shows some non-evidence-based indications of when the use of an interval training protocol may be more appropriate. If a patient is in a borderline status at some of these points it is recommended to let the patient decide which exercise protocol they would prefer. The patient could try both

\section{TABLE 3 Practical indications for considering the use of an interval training approach}

Interval training may be more appropriate when the patient presents with:

A severe airflow obstruction (FEV $1<40 \%$ pred)

A low exercise capacity (peak work rate $<60 \%$ pred)

A total time at a constant work rate test of $<10 \mathrm{~min}$

A marked oxygen desaturation during exercise $\left(\mathrm{SpO}_{2}<85 \%\right)$

An intolerable dyspnoea during continuous endurance training

FEV 1 : forced expiratory volume in $1 \mathrm{~s} ; \%$ pred: \% predicted; $\mathrm{SpO}_{2}$ : arterial oxygen saturation measured by pulse oximetry. modalities on the first days of a pulmonary rehabilitation programme and share their own opinion. Integrating the patient in the planning of their exercise programme may also improve their willingness to adhere to the intervention.

\section{Cycle-based versus walking-based endurance training}

Walking is one of the most important activities of daily living in patients with COPD. However, most endurance training programmes are based only on cycle endurance training. In addition to the higher costs and space requirement involving a treadmill in comparison to a cycle ergometer, another possible explanation for this fact could be that patients with COPD exhibit a greater ventilatory response during walking compared to cycling [31]. Thus, minimising dyspnoea sensations and the potential of oxygen desaturation during high intensity exercise are arguments in favour of providing cycling-based endurance training. However, walking-based endurance training programmes are also very effective in improving exercise capacity and quality of life in people with COPD [32, 33]. Compared to equipment-dependent training, such as cycle training, non-treadmill walking is an easily available training modality, particularly for those living in places with limited resources. Furthermore, exercising the patients' walking skills might be more effective to the patient than exercising cycling skills that are unlikely to be essential to everyday life. A recent study has even shown that supervised, progressive walking training resulted in a significantly larger increase in endurance walking capacity compared to supervised, progressive stationary cycle training [34]. Similar effects were found on peak walking and cycling capacity, endurance cycling capacity and health-related quality of life.

Since walking endurance capacity in patients with COPD is especially impaired, this could be the rationale for the implementation of walking-based endurance training to improve the patients walking capabilities.

Up-to-date detailed recommendations for prescribing walking training can rarely be found in the literature. A common 
reference point that was used in several studies [34, 35] was to set the walking speed at $\sim 80 \%$ of peak $V^{\prime} \mathrm{O}_{2}$, which was achieved in a shuttle walking test. To avoid the complex and time consuming procedure of a ergospirometry during the shuttle walk test, peak $V^{\prime} \mathrm{O}_{2}$ can be approximately derived by the following equation: peak $V^{\prime} \mathrm{O}_{2} \mathrm{~mL} \cdot \mathrm{min}^{-1} \cdot \mathrm{kg}^{-1}=$ $4.19+0.025 \times$ ISWT distance [36]. It has been shown that this intensity is feasible in most patients and is effective in improving exercise capacity [32]. If patients are not able to walk continuously for at least $10 \mathrm{~min}$ at the given speed, the intensity could be decreased stepwise by $\sim 10 \%$ until the patient is able to walk without taking a rest.

It is easy to set and control the proper walking speed when patients are exercising on a treadmill. If patients walk on the ground it is much more difficult to accurately stick to the individualised pace. Other methods such as using a metronome or listening to music, the rhythm of which is adjusted to the individual walking speed, might be useful $[35,37]$. Also a given track with distance marks could be helpful. If no supportive devices are available to determine walking speed, a perceived exertion on the Borg scale from 4 from 6 could be targeted. Furthermore, an interval approach could also be applied. To date, walking interval training has not been investigated. Due to practical reasons, longer interval walking periods of 1-2 min duration might be more appropriate.

\section{Oxygen supplementation during exercise}

The benefits of long-term oxygen therapy (LTOT) in patients with COPD associated with hypoxaemia are well known. In these patients, LTOT prolongs survival and reduces hospitalisations, as well as the risk of comorbidities [38, 39]. More recently, the usefulness of oxygen therapy in improving outcomes from pulmonary rehabilitation in patients with COPD has been evaluated in several studies. In general, a distinction must be made between immediate effects of oxygen on exercise performance and its usefulness in the exercisetraining component of pulmonary rehabilitation. As an adjunct to exercise training, supplemental oxygen therapy has been studied in patients who are severely hypoxaemic at rest or with exercise. The rationale for these studies is that supplemental oxygen therapy improves peripheral muscle oxygenation [40], dyspnoea [41] and exercise capacity [42] in patients with COPD and hypoxaemia, possibly allowing them to train at higher intensities. While the use of oxygen improves maximal exercise performance acutely in the laboratory, studies testing its effect in enhancing the exercise-training effects have produced inconsistent results. This may reflect differences in methodology among the studies, especially with regard to training workload [43]. However, the use of continuous supplemental oxygen for patients with COPD and severe resting hypoxaemia is clearly indicated and recommended as a part of routine clinical practice. Oxygen saturation measured by pulse oximetry $>90 \%$ and/or an arterial oxygen pressure $>55 \mathrm{mmHg}$ should be targeted [44].

The use of supplemental oxygen as an adjunct to exercise training could also be useful in patients who do not meet inclusion criteria for LTOT and do not experience exerciseinduced hypoxaemia. In a double-blinded randomised trial, patients without significant exercise-induced oxygen desaturation were randomised to receive either room air or oxygen during high-intensity exercise training [45]. Exercise performance improved significantly more in the group receiving oxygen. This improvement was accompanied by a reduction in respiratory rate.

The long-term effects when supplemental oxygen is discontinued and the effect on other outcomes such as health-related quality of life remain to be determined.

\section{Strength training}

Peripheral muscle dysfunction and muscle weakness are highly prevalent comorbidities of COPD, contributing to exercise intolerance and symptom intensity [46, 47]. It is assumed that resistance training can reverse peripheral muscle dysfunction and thereby reduce, at least in part, the burden of COPD impairment [48]. Resistance training as an adjunct to endurance is recommended in all patients especially those with peripheral muscle weakness. Because strength training has a greater potential to improve muscle mass and strength than endurance training $[49,50]$, a combination of these two exercise modalities is highly recommended. Strength training also provokes less dyspnoea during exercise, which most probably makes it easier to tolerate than aerobic training [51]. Therefore, a combination of resistance training with interval endurance training can be a useful alternative training strategy in patients severely restricted in their ability to perform endurance training due to marked ventilatory limitation.

A systematic review that included 18 randomised controlled trials showed consistent improvements in muscle strength despite a large variation of exercise characteristics, such as number of repetitions, exercise intensity or the method of strength training itself (e.g. conventional strength training machines, pulleys, free weights, Thera-Band; Hygenic Corporation, Akron, OH, USA) [52]. Therefore, the ideal exercise modalities for strength training in patients with COPD remain unknown. It appears that there is more than one mechanism to improve strength skills. Another important and clinically relevant finding of this article was that the effects of strength training may also be translated into meaningful changes in functional performance such as climbing stairs, standing up or arm elevation activities [52].

The ATS/ERS statement on pulmonary rehabilitation recommends performing two to four sets of six to 12 repetitions at intensities ranging from $50 \%$ to $85 \%$ of the one repetition maximum (1RM) 2-3 days per week (table 4) [53]. However, the principle of deriving strength training intensities from the $1 R M$ must be critically considered. Due to a large interindividual discrepancy and a wide range of variability of the number of possible repetitions at a certain percentage of the $1 R M$, the ideal resistance for many individuals may be over- or underestimated $[54,55]$. Therefore, the main focus could rather be on targeting local muscular exhaustion within the range of six to 12 repetitions (in other words determine a six to 12 repetition maximum) rather than reaching a certain percentage of the 1RM. Of course the patient must be instructed on this obligatory goal of achieving muscular exhaustion, otherwise they may not be willing to expose themselves to this exertion. An easy and very practical approach to determine optimal resistance for strength training is that the physiotherapist sets a training load so the patient is able to repeat an exercise six to 


\begin{tabular}{ll}
\hline TABLE 4 & $\begin{array}{l}\text { Practical recommendations for the implementation } \\
\text { of strength training }\end{array}$ \\
$\begin{array}{l}\text { Frequency } \\
\text { Objective }\end{array}$ & $\begin{array}{l}\text { 2-3 days } \text { week }^{-1} \\
\text { Targeting for local muscular exhaustion within a given number } \\
\text { of repetitions for major muscle groups of upper and lower } \\
\text { extremities }\end{array}$ \\
Mode & $\begin{array}{l}\text { Two to four sets of six to } 12 \text { repetitions } \\
\text { Intensity } \\
\text { Increase work load by 2-10\% if one to two repetitions over } \\
\text { the desired number are possible on two consecutive } \\
\text { training sessions } \\
\text { Moderate (1-2 s concentric and 1-2 s eccentric) }\end{array}$ \\
Speed & \\
\hline Data from [53]. &
\end{tabular}

maximally 12 times and has to stop due to muscle fatigue. With a little experience from the physiotherapist this is a very quick and useful approach that does not necessarily require determining the 1RM. However, it is recommended to use both upper and lower limb resistance weight training conducted with moderate speed at 1-2 s for both concentric and eccentric. When the subject can perform the current workload for one or two repetitions over the desired number of six to 12 repetitions on two consecutive training sessions it is recommended to apply a $2-10 \%$ increase in load [56].

\section{BEYOND "CONVENTIONAL" EXERCISE MODALITIES}

In recent years some additional exercise modalities beyond endurance and strength training have gained increasing interest. Due to the limited space of this article some of these new training components will only be briefly introduced.

\section{Inspiratory muscle training}

As a consequence of COPD, strength and endurance [57] of the diaphragm can also be reduced and contribute to hypercapnia, dyspnoea and reduced walking capacity [58]. Inspiratory muscle training may enhance the dysfunction of the diaphragm and improve some of its consequent burden. Various metaanalyses $[59,60]$ have shown that inspiratory muscle training can improve inspiratory muscle strength and endurance, as well as reduce dyspnoea. Scientific data on its benefits on functional exercise capacity and quality of life are less consistent, but evidence is emerging [60]. In patients suffering from inspiratory muscle weakness, defined as maximal inspiratory pressure (PImax) $<60 \mathrm{cmH}_{2} \mathrm{O}$, the addition of inspiratory muscle training to a general exercise training programme improved PImax and tended to improve exercise performance [61]. More studies that investigate inspiratory muscle training, especially in patients with inspiratory muscle weakness, are needed.

By far, the most commonly used inspiratory muscle training technique is the one of "threshold loading" devices (table 5). These devices generally have a spring-loaded valve requiring the patient to inhale strongly enough to open the valve and to breathe in against an individualised load. The optimal training intensity is still unknown but an initial resistance of $\geqslant 30 \%$ of PImax is recommended [60]. Resistance should then be increased stepwise, as tolerated. At present the optimal exercise duration is uncertain, most studies provided a total exercise time of

\begin{tabular}{ll} 
TABLE 5 & $\begin{array}{l}\text { Practical recommendations for the implementation } \\
\text { of inspiratory muscle training }(\mathrm{IMT})\end{array}$ \\
$\begin{array}{l}\text { Frequency } \\
\text { Objective }\end{array}$ & $\begin{array}{l}\text { To increase inspiratory muscle strength in patients with } \\
\text { inspiratory muscle weakness }\left(P \max <60 \mathrm{cmH}_{2} \mathrm{O}\right)\end{array}$ \\
$\begin{array}{l}\text { Mode } \\
\text { Intensity }\end{array}$ & $\begin{array}{l}\text { Initially } \geqslant 30 \% \text { of } \text { Plmax } \\
\text { Increase load as tolerated }\end{array}$ \\
Duration & $\begin{array}{l}\text { For example, using an interval approach with } 7 \times 2 \text { min of IMT } \\
\text { and } 1 \text { min of rest between each interval }\end{array}$ \\
\hline
\end{tabular}

PImax: maximal inspiratory pressure. Data from $[60,62]$

15-20 min per day [63]. It might be helpful to split inspiratory muscle training into two to three short exercise sessions per day.

\section{Neuromuscular electrical stimulation}

During neuromuscular electrical stimulation (NMES) the muscles get stimulated electronically via adhesive electrodes placed on the skin. As the metabolic response during a NMES session is significantly lower compared to a resistance exercise training session in patients with COPD [64], this technique may be particularly relevant to severely deconditioned or bedbound patients [65]. The most consistent finding of NMES training in COPD is a $20-30 \%$ gain in quadriceps strength as compared with control subjects [66, 67]. Most exercise protocols aim for the muscles of the thigh and the calf muscle. In NMES studies the duty cycle ranges between 2 to $10 \mathrm{~s}$ on and 4 to $50 \mathrm{~s}$ off for 20-60 min and one to two sessions per day 3-7 days per week (table 6) [68]. To set the initial stimulus the intensity will be increased until a visible strong muscle contraction occurs or to the maximum level of the patients' toleration. A novel finding is that it seems that there is a certain threshold for responders and non-responders to NMES. The gains in functional exercise capacity were heavily dependent on the ability of the patient to increase and sustain a progressively higher current intensity. The relationship between the change in walking test distance was characterised by a threshold of $10 \mathrm{~mA}$ increase from the beginning until the

\begin{tabular}{|c|c|}
\hline TABLE 6 & $\begin{array}{l}\text { Practical recommendations for the implementation } \\
\text { of neuromuscular electrical stimulation }\end{array}$ \\
\hline Frequency & 3-7 days $\cdot$ week $^{-1}$ \\
\hline Objective & $\begin{array}{l}\text { To increase peripheral muscle strength in severely } \\
\text { deconditioned or bed-bound patients }\end{array}$ \\
\hline Mode & $\begin{array}{l}\text { Pulse duration } 200-700 \mu \mathrm{s} \\
\text { Duty cycle } 2-10 \mathrm{~s} \text { on } / 4-50 \mathrm{~s} \text { off }\end{array}$ \\
\hline Intensity & $\begin{array}{l}\text { Increase intensity }(\mathrm{mA}) \text { until a visible muscle contraction } \\
\text { occurs or to the maximum tolerated level } \\
\text { Increase intensity whenever possible }\end{array}$ \\
\hline Duration & $\begin{array}{l}\text { One to two sessions per day, total exercise time per day } \\
20-60 \text { min }\end{array}$ \\
\hline
\end{tabular}

Data from [69]. 
end of a 6-week NMES intervention below which changes in exercise capacity with NMES were practically absent [69].

\section{Whole body vibration training}

During whole body vibration training subjects exercise on a vibrating platform that produces sinusoidal oscillations. At high intensities this vibration evokes muscle contractions on the entire flexor and extensor chain of muscles in the legs and all the way up to the trunk. Instead of voluntary muscle control like in common resistance training, the muscle contractions during high-intensity vibration training are caused by stretch reflexes [70]. The user has no direct influence on muscle activity itself and can only control body posture movement.

To date there is some evidence that whole body vibration training yields improvements of similar magnitude in regards of exercise capacity, muscle force and quality of life in comparison to conventional strength training [71]. Another study concluded that in patients with advanced COPD, whole body vibration training seems to be an effective and feasible exercise modality that may even enhance functional exercise capacity significantly more when performed in addition to endurance and strength training [72]. The underlying mechanisms are not yet investigated, but it is speculated that improvements in neuromuscular activation may play an important role. Table 7 provides some practical guidelines for the use of whole body vibration training.

\section{Breathing retraining (or breathing exercises)}

Breathing retraining is a simple approach aiming to alter respiratory muscle recruitment in order to reduce dyspnoea and improve respiratory muscle performance. Breathing retraining techniques described over time have mainly included pursed-lip breathing, diaphragmatic breathing and expiratory muscle strengthening. The improvements with breathing retraining discussed in the literature have included improvements in dyspnoea, work on breathing, ventilation, lung volume, functional performance and activities in daily living. However, many argue the improvements may not be due to breathing retraining alone but rather due to the adjunctive therapies such as medications, use of oxygen, and exercise training itself [73]. However, in a meta-analysis by

\begin{tabular}{ll}
\hline TABLE 7 & $\begin{array}{l}\text { Practical recommendations for the implementation } \\
\text { of whole body vibration training }\end{array}$ \\
\hline $\begin{array}{l}\text { Frequency } \\
\text { Objective }\end{array}$ & $\begin{array}{l}3 \text { days } \cdot \text { week }^{-1} \\
\text { To increase peripheral muscle strength and neuromuscular } \\
\text { activation, especially of lower limbs } \\
\text { Side-alternating or vertical vibration platform, peak-to-peak } \\
\text { displacement } \geqslant 4 \mathrm{~mm}\end{array}$ \\
Mode & $\begin{array}{l}\text { High intensities } \\
\text { Side-alternating vibration platforms: }>20 \mathrm{~Hz} \\
\text { Vertical vibration platforms: }<35 \mathrm{~Hz} \\
\text { Increase intensity by using additional weight, such as } \\
\text { dumbbells or a weighted backpack }\end{array}$ \\
Duration & Two to four sets of each $30-120 \mathrm{~s} \mathrm{per} \mathrm{exercise}$ \\
\hline Data from [73]. &
\end{tabular}

Holland et al. [74] it was concluded that breathing exercises improve functional exercise capacity in patients with COPD compared to no intervention despite the fact that there are no consistent effects on dyspnoea or health-related quality of life. Breathing exercises may be useful to improve exercise tolerance in selected individuals with COPD who are unable to undertake exercise training [74].

\section{Assessing the effectiveness of the exercise training programme}

Any pulmonary rehabilitation programme should include given outcome assessments, required for the objective evaluation of programme effectiveness, and of patient progress during the testing period. As currently practiced, pulmonary rehabilitation typically includes several different components, including not only exercise training but also education, nutritional and psychosocial support, and instruction in various respiratory and chest physiotherapy techniques. As an example, different outcomes to be assessed concerning their effectiveness after pulmonary rehabilitation include (but are not restricted to): overall exercise capacity (exercise tests), upper and lower limbs strength, symptoms (breathlessness and fatigue), health-related quality of life (specific questionnaires), and the improvement in physical activity performed in daily life not only in exercise tests.

\section{CONCLUSIONS}

In all stages of COPD, exercise training performed in the context of a pulmonary rehabilitation programme has been shown to be effective in a number of outcomes of patients with COPD, such as improved exercise tolerance, muscle strength, quality of life, and reduced dyspnoea and fatigue. Pulmonary rehabilitation is now a well-recognised therapy that should be available to all patients with symptomatic COPD, and exercise training is the cornerstone of a pulmonary rehabilitation programme. This article has provided specific details on the rationale of why and especially how to implement exercise training in patients with COPD, including the prescription of training mode, intensity and duration, as well as suggestions of guidelines for training progression.

\section{STATEMENT OF INTEREST}

Conflict of interest information can be found alongside the online version of this article at err.ersjournals.com

\section{REFERENCES}

1 Lacasse Y, Martin S, Lasserson TJ, et al. Meta-analysis of respiratory rehabilitation in chronic obstructive pulmonary disease. A Cochrane systematic review. Eura Medicophys 2007; 43: 475-485.

2 Ries AL, Bauldoff GS, Carlin BW, et al. Pulmonary Rehabilitation: Joint ACCP/AACVPR Evidence-Based Clinical Practice Guidelines. Chest 2007; 131: Suppl. 5, 4S-42S.

3 Global Strategy for the Diagnosis, Management and Prevention of COPD. Global Initiative for Chronic Obstructive Lung Disease (GOLD). 2013. Available from www.goldcopd.org Date last accessed: February 10, 2013.

4 Eliason G, Abdel-Halim S, Arvidsson B, et al. Physical performance and muscular characteristics in different stages of COPD. Scand J Med Sci Sports 2009; 19: 865-870. 
5 Puente-Maestu L, Garcia de Pedro J, Martinez-Abad Y, et al. Dyspnea, ventilatory pattern, and changes in dynamic hyperinflation related to the intensity of constant work rate exercise in COPD. Chest 2005; 128: 651-656.

6 Singh SJ, Morgan MD, Scott S, et al. Development of a shuttle walking test of disability in patients with chronic airways obstruction. Thorax 1992; 47: 1019-1024.

7 American Thoracic Society, American College of Chest Physicians. ATS/ACCP statement on cardiopulmonary exercise testing. Am J Respir Crit Care Med 2003; 167: 211-277.

8 Myers J, Buchanan N, Walsh D, et al. Comparison of the ramp versus standard exercise protocols. J Am Coll Cardiol 1991; 17: 1334-1342.

9 Oga T, Nishimura K, Tsukino M, et al. The effects of oxitropium bromide on exercise performance in patients with stable chronic obstructive pulmonary disease. A comparison of three different exercise tests. Am J Respir Crit Care Med 2000; 161: 1897-1901.

10 Pitta F, Troosters T, Spruit MA, et al. Characteristics of physical activities in daily life in chronic obstructive pulmonary disease. Am J Respir Crit Care Med 2005; 171: 972-927.

11 Garcia-Talavera I, Garcia $\mathrm{CH}$, Macario $\mathrm{CC}$, et al. Time to desaturation in the 6-min walking distance test predicts 24-hour oximetry in COPD patients with a $\mathrm{PO}_{2}$ between 60 and $70 \mathrm{mmHg}$. Respir Med 2008; 102: 1026-1032.

12 ATS Committee on Proficiency Standards for Clinical Pulmonary Function Laboratories. ATS statement: guidelines for the sixminute walk test. Am J Respir Crit Care Med 2002; 166: 111-117.

13 Hill K, Dolmage TE, Woon L, et al. A simple method to derive speed for the endurance shuttle walk test. Respir Med 2012; 106: $1665-1670$.

14 Revill SM, Morgan MD, Singh SJ, et al. The endurance shuttle walk: a new field test for the assessment of endurance capacity in chronic obstructive pulmonary disease. Thorax 1999; 54: 213-222.

15 Canuto FF, Rocco CC, de Andrade DV, et al. Neurophysiological comparison between the sit-to-stand test with the 6-minute walk test in individuals with COPD. Electromyogr Clin Neurophysiol 2010; 50: 47-53.

16 Ozalevli S, Ozden A, Itil O, et al. Comparison of the sit-to-stand test with 6 min walk test in patients with chronic obstructive pulmonary disease. Respir Med 2007; 101: 286-293.

17 Puhan MA, Siebeling L, Zoller M. Simple functional performance tests and mortality in COPD. Eur Respir J 2013 [In press DOI: 10.1183/09031936.00131612].

18 Man WD, Kemp P, Moxham J, et al. Skeletal muscle dysfunction in COPD: clinical and laboratory observations. Clin Sci (Lond) 2009; 117: 251-264.

19 Mathiowetz V, Kashman N, Volland G, et al. Grip and pinch strength: normative data for adults. Arch Phys Med Rehabil 1985; 66: 69-74.

20 Andrews AW, Thomas MW, Bohannon RW. Normative values for isometric muscle force measurements obtained with hand-held dynamometers. Phys Ther 1996; 76: 248-259.

21 Vogiatzis I, Terzis G, Nanas S, et al. Skeletal muscle adaptations to interval training in patients with advanced COPD. Chest 2005; 128 : 3838-3845.

22 Casaburi R, Porszasz J, Burns MR, et al. Physiologic benefits of exercise training in rehabilitation of patients with severe chronic obstructive pulmonary disease. Am J Respir Crit Care Med 1997; 155: 1541-1551.

23 Maltais F, LeBlanc $\mathrm{P}$, Jobin $\mathrm{J}$, et al. Intensity of training and physiologic adaptation in patients with chronic obstructive pulmonary disease. Am J Respir Crit Care Med 1997; 155: 555-561.

24 Beauchamp MK, Nonoyama M, Goldstein RS, et al. Interval versus continuous training in individuals with chronic obstructive pulmonary disease - a systematic review. Thorax 2010; 65: 157-164.
25 Vogiatzis I, Terzis G, Stratakos G, et al. Effect of pulmonary rehabilitation on peripheral muscle fiber remodeling in patients with COPD in GOLD stages II to IV. Chest 2011; 140: 744-752.

26 Puhan MA, Busching G, Schunemann HJ, et al. Interval versus continuous high-intensity exercise in chronic obstructive pulmonary disease: a randomized trial. Ann Intern Med 2006; 145: 816-825.

27 Gloeckl R, Halle M, Kenn K. Interval versus continuous training in lung transplant candidates: a randomized trial. J Heart Lung Transplant 2012; 31: 934-941.

28 Vogiatzis I, Nanas S, Kastanakis E, et al. Dynamic hyperinflation and tolerance to interval exercise in patients with advanced COPD. Eur Respir J 2004; 24: 385-390.

29 Vogiatzis I, Nanas S, Roussos C. Interval training as an alternative modality to continuous exercise in patients with COPD. Eur Respir J 2002; 20: 12-19.

30 Kortianou EA, Nasis IG, Spetsioti ST, et al. Effectiveness of interval exercise training in patients with COPD. Cardiopulm Phys Ther J 2010; 21: 12-19.

31 Mahler D, Gifford AH, Waterman LA, et al. Mechanism of greater oxygen desaturation during walking compared with cycling in COPD. Chest 2011; 140: 351-358.

32 Liu WT, Wang CH, Lin HC, et al. Efficacy of a cell phone-based exercise programme for COPD. Eur Respir J 2008; 32: 651-659.

33 Hernandez MT, Rubio TM, Ruiz FO, et al. Results of a home-based training program for patients with COPD. Chest 2000; 118: 106-114.

34 Leung RW, Alison JA, McKeough ZJ, et al. Ground walk training improves functional exercise capacity more than cycle training in people with chronic obstructive pulmonary disease (COPD): a randomised trial. J Physiother 2010; 56: 105-112.

35 Ho CF, Maa SH, Shyu YI, et al. Effectiveness of paced walking to music at home for patients with COPD. COPD 2012; 9: 447-457.

36 Singh SJ, Morgan MD, Hardman AE, et al. Comparison of oxygen uptake during a conventional treadmill test and the shuttle walking test in chronic airflow limitation. Eur Respir J 1994; 7: 2016-2020.

37 Pomidori L, Contoli M, Mandolesi G, et al. A simple method for home exercise training in patients with chronic obstructive pulmonary disease: one-year study. J Cardiopulm Rehabil Prev 2012; 32: 53-57.

38 Long term domiciliary oxygen therapy in chronic hypoxic cor pulmonale complicating chronic bronchitis and emphysema. Report of the Medical Research Council Working Party. Lancet 1981; 1: 681-686.

39 Continuous or nocturnal oxygen therapy in hypoxemic chronic obstructive lung disease: a clinical trial. Nocturnal Oxygen Therapy Trial Group. Ann Intern Med 1980; 93: 391-398.

40 Vogiatzis I, Athanasopoulos D, Stratakos G, et al. Exercise-induced skeletal muscle deoxygenation in O-supplemented COPD patients. Scand J Med Sci Sports 2009; 19: 364-372.

41 Woodcock AA, Gross ER, Geddes DM. Oxygen relieves breathlessness in "pink puffers". Lancet 1981; 1: 907-909.

42 O'Donnell DE, D'Arsigny C, Webb KA. Effects of hyperoxia on ventilatory limitation during exercise in advanced chronic obstructive pulmonary disease. Am J Respir Crit Care Med 2001; 163: 892-898.

43 Puhan MA, Schunemann HJ, Frey M, et al. Value of supplemental interventions to enhance the effectiveness of physical exercise during respiratory rehabilitation in COPD patients. A systematic review. Respir Res 2004; 5: 25.

44 Guell Rous R. Long-term oxygen therapy: are we prescribing appropriately? Int J Chron Obstruct Pulmon Dis 2008; 3: 231-237.

45 Emtner M, Porszasz J, Burns M, et al. Benefits of supplemental oxygen in exercise training in nonhypoxemic chronic obstructive pulmonary disease patients. Am J Respir Crit Care Med 2003; 168: 1034-1042.

46 Gosselink R, Troosters T, Decramer M. Peripheral muscle weakness contributes to exercise limitation in COPD. Am J Respir Crit Care Med 1996; 153: 976-980. 
47 Hamilton AL, Killian KJ, Summers E, et al. Muscle strength, symptom intensity, and exercise capacity in patients with cardiorespiratory disorders. Am J Respir Crit Care Med 1995; 152: 2021-2031.

48 Troosters T, Casaburi R, Gosselink R, et al. Pulmonary rehabilitation in chronic obstructive pulmonary disease. Am J Respir Crit Care Med 2005; 172: 19-38

49 Spruit MA, Gosselink R, Troosters T, et al. Resistance versus endurance training in patients with COPD and peripheral muscle weakness. Eur Respir J 2002; 19: 1072-1078.

50 Ortega F, Toral J, Cejudo P, et al. Comparison of effects of strength and endurance training in patients with chronic obstructive pulmonary disease. Am J Respir Crit Care Med 2002; 166: 669-674.

51 Simpson K, Killian K, McCartney N, et al. Randomised controlled trial of weightlifting exercise in patients with chronic airflow limitation. Thorax 1992; 47: 70-75.

52 O'Shea SD, Taylor NF, Paratz JD. Progressive resistance exercise improves muscle strength and may improve elements of performance of daily activities for people with COPD: a systematic review. Chest 2009; 136: 1269-1283.

53 Nici L, ZuWallack R, Wouters E, et al. On pulmonary rehabilitation and the flight of the bumblebee: the ATS/ERS Statement on Pulmonary Rehabilitation. Eur Respir J 2006; 28: 461-462.

54 Walker M, Sussman D, Tamburello D, et al. Relationship between maximum strength and relative endurance for the empty can exercise. J Sport Rehabil 2003; 12: 31-38.

55 Kraemer WJ, Ratamess NA. Fundamentals of resistance training: progression and exercise prescription. Med Sci Sports Exerc 2004; 36: 674-688.

56 Kraemer WJ, Adams K, Cafarelli E, et al. American College of Sports Medicine position stand. Progression models in resistance training for healthy adults. Med Sci Sports Exerc 2002; 34: 364-380.

57 Rochester DF, Braun NM. Determinants of maximal inspiratory pressure in chronic obstructive pulmonary disease. Am Rev Respir Dis 1985; 132: 42-47.

58 Wijkstra PJ, Ten Vergert EM, van der Mark TW, et al. Relation of lung function, maximal inspiratory pressure, dyspnoea, and quality of life with exercise capacity in patients with chronic obstructive pulmonary disease. Thorax 1994; 49: 468-472.

59 Geddes EL, O'Brien K, Reid WD, et al. Inspiratory muscle training in adults with chronic obstructive pulmonary disease: an update of a systematic review. Respir Med 2008; 102: 1715-1729.

60 Gosselink R, De Vos J, van den Heuvel SP, et al. Impact of inspiratory muscle training in patients with COPD: what is the evidence? Eur Respir J 2011; 37: 416-425.
61 Lotters F, van Tol B, Kwakkel G, et al. Effects of controlled inspiratory muscle training in patients with COPD: a metaanalysis. Eur Respir J 2002; 20: 570-576.

62 Hill K, Jenkins SC, Philippe DL, et al. High-intensity inspiratory muscle training in COPD. Eur Respir J 2006; 27: 1119-1128.

63 Langer D, Hendriks E, Burtin C, et al. A clinical practice guideline for physiotherapists treating patients with chronic obstructive pulmonary disease based on a systematic review of available evidence. Clin Rehabil 2009; 23: 445-462.

64 Sillen MJ, Janssen PP, Akkermans MA, et al. The metabolic response during resistance training and neuromuscular electrical stimulation (NMES) in patients with COPD, a pilot study. Respir Med 2008; 102: 786-789.

65 Vivodtzev I, Lacasse Y, Maltais F. Neuromuscular electrical stimulation of the lower limbs in patients with chronic obstructive pulmonary disease. J Cardiopulm Rehabil Prev 2008; 28: 79-91.

66 Zanotti E, Felicetti G, Maini M, et al. Peripheral muscle strength training in bed-bound patients with COPD receiving mechanical ventilation: effect of electrical stimulation. Chest 2003; 124: 292-296.

67 Neder JA, Sword D, Ward SA, et al. Home based neuromuscular electrical stimulation as a new rehabilitative strategy for severely disabled patients with chronic obstructive pulmonary disease (COPD). Thorax 2002; 57: 333-337.

68 Sillen MJ, Speksnijder CM, Eterman RM, et al. Effects of neuromuscular electrical stimulation of muscles of ambulation in patients with chronic heart failure or COPD: a systematic review of the English-language literature. Chest 2009; 136: 44-61.

69 Vivodtzev I, Debigare R, Gagnon P, et al. Functional and muscular effects of neuromuscular electrical stimulation in patients with severe COPD: a randomized clinical trial. Chest 2012; 141: 716-725.

70 Cardinale M, Lim J. Electromyography activity of vastus lateralis muscle during whole-body vibrations of different frequencies. J Strength Cond Res 2003; 17: 621-624.

71 Salhi BJ, van Meerbeeck J, Joos GF, et al. Effects of whole body vibration in patients with COPD: a randomized study. Am J Respir Crit Care Med 2011; 183: A3968.

72 Gloeckl R, Heinzelmann I, Baeuerle S, et al. Effects of whole body vibration in patients with chronic obstructive pulmonary disease a randomized controlled trial. Respir Med 2012; 106: 75-83.

73 Hillegass EA. Breathing retraining for individuals with chronic obstructive pulmonary disease - a role for clinicians. Chron Respir Dis 2009; 6: 43-44.

74 Holland AE, Hill CJ, Jones AY, et al. Breathing exercises for chronic obstructive pulmonary disease. Cochrane Database Syst Rev 2012; 10: CD008250. 